\title{
Prepubertal stature and blood pressure in early old age
}

\author{
Scott M Montgomery, Lee R Berney, David Blane
}

\begin{abstract}
Aims-To test the hypothesis that childhood growth rate is a marker for formation of control mechanisms that influence blood pressure in early old age.

Methods-Data are from a sample of 149 (74 male) members of Sir John Boyd Orr's survey of British families conducted between 1937 and 1939. Measured heights were collected between ages 5 and 8 years, and in early old age between 1997 and 1998. Multiple linear regression investigated the relations of blood pressure with age and sex standardised childhood height with adjustment for potential confounding factors, including adult height. Inclusion of both childhood and adult heights in the same model was used to estimate growth, as measures of childhood height are relative to adult height.

Results-Mean blood pressures in early old age for those in the shortest childhood height fifth were 167.8 and $76.3 \mathrm{~mm} \mathrm{Hg}$ for systolic blood pressure and pulse pressure, respectively. For the tallest fifth they were 150.8 and $63.7 \mathrm{~mm} \mathrm{Hg}$, respectively. After adjustment for potential confounding factors including adult height, the mean increase for the shortest childhood height fifth compared with the tallest was $28.5 \mathrm{~mm} \mathrm{Hg}$ for systolic pressure $(p=0.015)$ and $22.8 \mathrm{~mm} \mathrm{Hg}(\mathrm{p}=0.010)$ for pulse pressure. The relations of blood pressure with adult height were not statistically significant in the adjusted models. Conclusion-Prepubertal growth rate is associated with the formation of mechanisms associated with the control of blood pressure in later life.

(Arch Dis Child 2000;82:358-363)
\end{abstract}

Keywords: blood pressure; growth; height; old age

Inverse relations between stature and blood pressure have previously been reported for both children ${ }^{1}$ and adults. ${ }^{2}$ Shorter adults are at greater risk of death from cardiovascular causes and it has been suggested that body size has a direct effect on cardiovascular function. ${ }^{3}$ Slower growth in childhood may result from adversity ${ }^{4}$ extending into adult life ${ }^{5}$ and accumulated risks associated with this disadvantage may influence blood pressure. As an alternative, but not mutually exclusive explanation for the relation of height with blood pressure, we hypothesise that height is a marker for factors that influence both growth rate and the formation of blood pressure control mechanisms.
We used longitudinal data to investigate the relations of growth in childhood with blood pressure in early old age and with pulse pressure, as a marker of arterial compliance. Old age is of particular interest in examining blood pressure, as this is a time of increased risk of cardiovascular morbidity and mortality, because blood pressure increases with age, ${ }^{6}$ possibly amplifying the effects of early exposures. To model growth, statistical models used childhood height (standardised for age and sex) adjusted for fully attained adult height. We tested the hypothesis: if early adversity influences mechanisms involved in the control of blood pressure, the relation with blood pressure should be stronger for childhood growth than it is for adult height. To take into account the effect of continuity of disadvantage and later-life risk factors, adjustment was performed for potential material and psychosocial risk factors accumulated over life.

Psychosocial stress has been shown to slow childhood growth rate $^{47}$ and also to result in poorer control of the stress response, ${ }^{8}$ possibly resulting in greater vulnerability to stress throughout life. Therefore, slower growing children (as an indicator of poorer control of the stress response) may be more vulnerable to high blood pressure if exposed to chronic psychosocial stress in adult life, such as working for long periods in jobs with low decision latitude. ${ }^{10-12}$

Subjects and methods

The data used here are available from the ESRC Data Archive and have been described in detail elsewhere. ${ }^{13-17}$

\section{SUBJECTS}

The subjects, when children, were among the participants of a survey of 1352 families living in 16 locations in Great Britain. Conducted between 1937 and 1939, this survey collected a range of social, dietary, health, and anthropometric data. ${ }^{13-15}$ Surviving participants were traced $^{15}$ and a random stratified sample of some 300 were surveyed in early old age between 1997 and $1998 .{ }^{14}$ Physiological and anthropometric measures were taken and information was collected about current health and social circumstances. Retrospective occupational and residential histories were recorded using the life grid method. ${ }^{17}$

Details of the representativeness of the sample have been published previously ${ }^{14}{ }^{17}$ : the social class distribution of subjects' fathers resembles the class distribution of young adult males at the 1931 decennial census; the sociodemographic characteristics of the subjects in email: smm@rfhsm.ac.uk

Accepted 4 February 2000 
early old age resemble those aged $65-74$ years in the British population at the 1991 decennial census. ${ }^{14}$

To minimise confounding caused by age related variation in growth velocity, the sample analysed here was restricted to include only the 149 cohort members who were assessed during the period of linear growth between ages 5 and 8 years (median age 6 years). Their median age at adult interview was 66 years with a range of 63-76 years. Despite broad representativeness, those included in the analysis tended to be of higher socioeconomic class than the general population, with $57 \%$ of subjects in a nonmanual social class compared with $49 \%$ of the general population aged 65-74 years, as indicated by decenial census data. ${ }^{18}$

\section{MEASURES}

Anthropometric measures

Heights and weights were measured at the interviews conducted during childhood and in early old age. Childhood heights have been age and sex standardised using standard deviation units ( $Z$ scores). ${ }^{15}$ This internal standardisation was derived, as a reference standard for prewar children is not available. ${ }^{19}$ Adult heights were also sex standardised using standard deviation units. Both heights were divided into fifths of their standardised distributions as previous research has identified children in the shortest age standardised height fifth as more likely to have experienced adversity ${ }^{4}$ and to be at a greater risk of subsequent disadvantage. ${ }^{5}$ Body mass index (BMI), divided into fifths of the distribution, was calculated for the childhood and adult heights and weights.

Health and material circumstances in childhood A medical examination in childhood recorded the presence of the following symptoms, signs, and diagnoses: otitis media, bronchitis, angular stomatitis, and knock knees. ${ }^{15}$ These symptoms, signs, and diagnoses were chosen as being indicative of poorer material circumstances and a combined score was created.

The weekly household expenditure on food in pennies was recorded prospectively when the cohort members were children. This was divided by the total number of people living in the household and the resulting figure was divided into fifths of its distribution. Household crowding used the person per room ratio divided into fifths. The social class based on occupation of the cohort member's father was reclassified using the 1991 Registrar General's schema. Unlike contemporaneous classifications, the 1991 schema allows class III to be subdivided into manual and non-manual groups. The classification was dichotomised into manual and non-manual classes.

Accumulated exposures throughout adult life A life grid interview, conducted in early old age, collected information about events and circumstances from childhood to the time of the interview. The life grid technique uses temporal inter-relations of events and circumstances to improve recall accuracy and reduce bias. $^{17}$
Three questions were used to quantify decision latitude at work: (1) Were you given the opportunity to make decisions about your work? (2) Would you say, overall, the work was varied or repetitive? (3) Were you able to take breaks when you wanted? If questions 1 and 3 were answered as "no" and 2 "repetitive", then the job was designated as having low decision latitude. The total number of years in a job with low decision latitude was calculated. The distribution for those with any low decision latitude job was divided into quarters and those who never had such jobs formed the fifth category. A similar variable for lifetime smoking habit was created, with one category for never smokers and the total numbers of years of smoking divided into quarters of its distribution. As a measure of material and cultural circumstances, the Registrar General's social class based on last main occupation was used. This was dichotomised into manual and non-manual classes.

\section{Health and material circumstances in early old} age

Systolic and diastolic blood pressure were measured using electrical sphygmomanometers in $\mathrm{mm} \mathrm{Hg}$. Pulse pressure was calculated by subtracting the value of diastolic from systolic blood pressure. During the interview it was established whether respondents were currently taking antihypertension medication. Current smoking status was also established at this time. Ex-smokers were identified using the previously discussed smoking variable which recorded number of years as a smoker. Whether or not the household had access to a motor car was recorded and these data were used in analysis as a binary variable. The current number of state benefits received was used as an additional measure of material circumstances. This variable has values for, none, one, two, and three or more benefits. Age at interview was divided into categories to accommodate the distribution: under 65, $65-67$, and 68 years or over.

\section{STATISTICAL METHODS}

The associations of mean systolic blood pressure, diastolic blood pressure, and pulse pressure with childhood and adult standardised height fifths were assessed. The relative strength of association of standardised height in childhood and early old age was examined using multiple linear regression with forced entry. With the exception of the three measures of blood pressure, all variables were modelled as binary dummies because of potential non-linearity in their relation with blood pressure.

Multiple linear regression was used to assess the relations of the two height measures (in separate models) with the three measures of blood pressure with adjustment for sex, age at interview, BMI, and current use of antihypertension medication. These analyses were repeated with the additional inclusion of the potential confounding factors associated with the lifetime accumulation of risk. The childhood factors are: the childhood symptom 
score, childhood BMI, family expenditure on food, household crowding, and father's class. The measures of accumulated exposures over life are: number of years smoking, and number of years in a job with low decision latitude. The factors for early old age are: number of benefits being received, smoking status, car ownership, and social class.

An additional set of analyses included, in addition to all other potential confounding factors, both adult and childhood heights in the same model to assess their independence from each other.

An interaction term was used to investigate slower growth as a marker for greater vulnerability to the subsequent psychosocial stress associated with low decision latitude at work. The interaction of being in the shortest childhood height fifth, with the greatest accumulation of working in a low decision latitude job, was investigated in models adjusted for all potential confounding factors, as well as the main interaction effects (childhood height and decision latitude).
All analyses were performed using SPSS software (version 8.0.2).

\section{Results}

Data for 74 men and 75 women were analysed, with mean blood pressures slightly higher for men, but this difference did not achieve statistical significance for any measure of blood pressure. Among men, the mean values for systolic blood pressure, diastolic blood pressure, and pulse pressure were 159.8 (SD 25.8) $\mathrm{mm} \mathrm{Hg}, 89.0$ (10.6) $\mathrm{mm} \mathrm{Hg}$, and 70.8 (19.0) $\mathrm{mm} \mathrm{Hg}$, respectively. For women the mean pressures were 154.7 (22.2) $\mathrm{mm} \mathrm{Hg}, 86.4$ (11.5) $\mathrm{mm} \mathrm{Hg}$, and 68.3 (16.8) $\mathrm{mm} \mathrm{Hg}$, respectively. To enhance statistical power and because of the lack of statistically significant differences in any of the analyses performed, men and women were modelled together, but with adjustment using the sex variable.

Age and sex standardised height in childhood shows an inverse relation with systolic blood pressure and pulse pressure, but not diastolic blood pressure in early old age (tables 1,2 , and

Table 1 Systolic blood pressure in early old age by childhood and adult heights

\begin{tabular}{|c|c|c|c|c|c|c|c|c|}
\hline & \multicolumn{2}{|l|}{$A$} & \multicolumn{2}{|l|}{$B$} & \multicolumn{2}{|l|}{$C$} & \multicolumn{2}{|l|}{$D$} \\
\hline & $n$ & Mean $(S D)$ & $\begin{array}{l}\text { Mean difference } \\
(95 \% \mathrm{CI})\end{array}$ & $p$ value & $\begin{array}{l}\text { Mean difference } \\
(95 \% \mathrm{CI})\end{array}$ & $p$ value & $\begin{array}{l}\text { Mean difference } \\
(95 \% \mathrm{CI})\end{array}$ & $p$ value \\
\hline \multicolumn{9}{|c|}{ Childhood height } \\
\hline 1 & 29 & $167.8(22.7)$ & $18.1(6.1,30.2)$ & 0.004 & $29.0(14.9,43.1)$ & 0.000 & $28.5(5.6,51.5)$ & 0.015 \\
\hline 2 & 30 & $156.9(23.6)$ & $6.1(-5.9,18.1)$ & 0.316 & $14.6(1.1,28.2)$ & 0.035 & $13.9(-5.3,33.1)$ & 0.155 \\
\hline 3 & 30 & $158.1(25.2)$ & $7.9(-4.3,20.1)$ & 0.202 & $13.8(-0.7,28.3)$ & 0.062 & $12.3(-7.8,32.4)$ & 0.227 \\
\hline 4 & 30 & $153.1(21.6)$ & $2.4(-9.5,14.2)$ & 0.694 & $11.8(-1.4,25.0)$ & 0.080 & $10.2(-7.7,28.2)$ & 0.260 \\
\hline 5 & 30 & $150.8(22.4)$ & 0.0 & & 0.0 & & 0.0 & \\
\hline Total & 149 & $157.3(23.5)$ & & & & & & \\
\hline \multicolumn{9}{|c|}{ Adult height } \\
\hline 1 & 30 & $162.0(20.7)$ & $13.5(1.3,25.8)$ & 0.031 & $19.3(5.8,32.9)$ & 0.006 & $0.7(-20.0,21.5)$ & 0.943 \\
\hline 2 & 29 & $159.4(19.6)$ & $11.8(-0.8,24.4)$ & 0.066 & $15.1(1.7,28.4)$ & 0.027 & $1.2(-17.6,20.0)$ & 0.899 \\
\hline 3 & 31 & $160.4(26.2)$ & $11.2(-1.3,23.8)$ & 0.076 & $12.8(-0.2,25.8)$ & 0.054 & $2.8(-14.4,20.0)$ & 0.749 \\
\hline 4 & 29 & $157.7(25.9)$ & $8.7(-3.8,21.2)$ & 0.171 & $10.7(-2.4,23.7)$ & 0.109 & $3.8(-12.0,19.6)$ & 0.635 \\
\hline 5 & 30 & $146.9(22.8)$ & 0.0 & & & & & \\
\hline Total & 149 & $157.3(23.5)$ & & & & & & \\
\hline
\end{tabular}

A. Mean pressure in $\mathrm{mm} \mathrm{Hg}$ with standard deviation by childhood and adult height fifths

B. Mean difference in pressure compared with tallest fifth using multiple linear regression, adjusted for sex, age at interview, BMI, and use of antihypertension medication. Childhood and adult heights are modelled separately.

C. As model B, but with additional adjustment for childhood symptom score, childhood BMI, family expenditure on food, household crowding, father's class, number of years smoking, number of years in a job with low decision latitude, number of benefits being received, smoking status, car ownership, and social class. Childhood and adult heights are modelled separately.

D. As model C, but childhood and adult heights are included in the same model.

Table 2 Diastolic blood pressure in early old age by childhood and adult heights

\begin{tabular}{|c|c|c|c|c|c|c|c|c|}
\hline & \multicolumn{2}{|l|}{$A$} & \multicolumn{2}{|l|}{$B$} & \multicolumn{2}{|l|}{$C$} & \multicolumn{2}{|l|}{$D$} \\
\hline & $n$ & Mean (SD) & $\begin{array}{l}\text { Mean difference } \\
(95 \% \mathrm{CI})\end{array}$ & $p$ value & $\begin{array}{l}\text { Mean difference } \\
(95 \% \mathrm{CI})\end{array}$ & $p$ value & $\begin{array}{l}\text { Mean difference } \\
(95 \% \mathrm{CI})\end{array}$ & $p$ value \\
\hline \multicolumn{9}{|c|}{ Childhood height } \\
\hline 1 & 29 & $91.5(9.5)$ & $4.5(-1.2,10.1)$ & 0.124 & $8.1(0.8,15.4)$ & 0.029 & $5.7(-6.1,17.5)$ & 0.343 \\
\hline 2 & 30 & $85.6(12.7)$ & $-1.9(-7.6,3.7)$ & 0.502 & $1.7(-5.3,8.7)$ & 0.637 & $-0.3(-10.2,9.5)$ & 0.947 \\
\hline 3 & 30 & 90.2 (11.4) & $2.3(-3.5,8.0)$ & 0.439 & $6.2(-1.3,13.7)$ & 0.104 & $3.6(-6.7,14.0)$ & 0.490 \\
\hline 4 & 30 & $84.4(8.5)$ & $-3.1(-8.7,2.5)$ & 0.279 & $1.7(-5.1,8.5)$ & 0.628 & $-0.4(-9.5,8.9)$ & 0.939 \\
\hline 5 & 30 & $87.1(11.9)$ & 0.0 & & 0.0 & & 0.0 & \\
\hline Total & 149 & $87.7(11.1)$ & & & & & & \\
\hline \multicolumn{9}{|c|}{ Adult height } \\
\hline 1 & 30 & $89.1(12.0)$ & $4.1(-1.7,9.9)$ & 0.167 & $6.0(-0.8,12.9)$ & 0.085 & $2.6(-8.1,13.2)$ & 0.633 \\
\hline 2 & 29 & $88.3(8.6)$ & $2.8(-3.1,8.8)$ & 0.348 & $4.3(-2.5,11.0)$ & 0.211 & $2.4(-7.3,12.0)$ & 0.629 \\
\hline 3 & 31 & $89.9(11.6)$ & $3.9(-2.1,9.8)$ & 0.203 & $6.0(-0.6,12.5)$ & 0.076 & $4.2(-4.7,13.0)$ & 0.353 \\
\hline 4 & 29 & $86.9(10.1)$ & $1.0(-4.9,6.9)$ & 0.741 & $2.8(-3.8,9.4)$ & 0.401 & $1.9(-6.2,10.1)$ & 0.635 \\
\hline 5 & 30 & $84.4(12.5)$ & 0.0 & & 0.0 & & 0.0 & \\
\hline Total & 149 & $87.7(11.1)$ & & & & & & \\
\hline
\end{tabular}

A. Mean pressure in $\mathrm{mm} \mathrm{Hg}$ with standard deviation by childhood and adult height fifths

B. Mean difference in pressure compared with tallest fifth using multiple linear regression, adjusted for sex, age at interview, BMI, and use of antihypertension medication. Childhood and adult heights are modelled separately.

C. As model B, but with additional adjustment for childhood symptom score, childhood BMI, family expenditure on food, household crowding, father's class, number of years smoking, number of years in a job with low decision latitude, number of benefits being received, smoking status, car ownership, and social class. Childhood and adult heights are modelled separately.

D. As model C, but childhood and adult heights are included in the same model. 
Table 3 Pulse pressure in early old age by childhood and adult heights

\begin{tabular}{|c|c|c|c|c|c|c|c|c|}
\hline & \multicolumn{2}{|l|}{$A$} & \multicolumn{2}{|l|}{$B$} & \multicolumn{2}{|l|}{$C$} & \multicolumn{2}{|l|}{$D$} \\
\hline & $n$ & Mean (SD) & $\begin{array}{l}\text { Mean difference } \\
(95 \% \mathrm{CI})\end{array}$ & $p$ value & $\begin{array}{l}\text { Mean difference } \\
(95 \% \mathrm{CI})\end{array}$ & $p$ value & $\begin{array}{l}\text { Mean difference } \\
(95 \% \mathrm{CI})\end{array}$ & $p$ value \\
\hline \multicolumn{9}{|c|}{ Childhood height } \\
\hline 1 & 29 & $76.3(19.7)$ & $13.7(4.4,22.9)$ & 0.004 & $20.9(10.3,31.5)$ & 0.000 & $22.8(5.6,40.1)$ & 0.010 \\
\hline 2 & 30 & $71.3(17.9)$ & $8.0(-1.2,17.2)$ & 0.086 & $13.0(2.8,23.2)$ & 0.013 & $14.2(-0.2,28.6)$ & 0.054 \\
\hline 3 & 30 & $68.0(18.0)$ & $5.7(-3.7,15.0)$ & 0.233 & $7.6(-3.3,18.5)$ & 0.168 & $8.7(-6.4,23.8)$ & 0.256 \\
\hline 4 & 30 & $68.8(16.6)$ & $5.5(-3.7,14.6)$ & 0.238 & $10.1(0.2,20.1)$ & 0.047 & $10.6(-2.9,24.1)$ & 0.122 \\
\hline 5 & 30 & $63.7(16.0)$ & 0.0 & & 0.0 & & 0.0 & \\
\hline Total & 149 & $69.6(17.9)$ & & & & & & \\
\hline \multicolumn{9}{|c|}{ Adult height } \\
\hline 1 & 30 & $72.8(16.0)$ & $9.4(0.1,18.8)$ & 0.048 & $13.3(3.1,23.6)$ & 0.011 & $-1.8(-17.4,13.8)$ & 0.817 \\
\hline 2 & 29 & $71.2(16.1)$ & $9.0(-0.7,18.6)$ & 0.068 & $10.8(0.7,20.8)$ & 0.036 & $-1.2(-15.2,13.0)$ & 0.872 \\
\hline 3 & 31 & $70.5(19.6)$ & $7.4(-2.2,17.0)$ & 0.131 & $6.8(-3.0,16.7)$ & 0.171 & $-1.3(-14.3,11.6)$ & 0.833 \\
\hline 4 & 29 & $70.8(20.2)$ & $7.7(-1.8,17.2)$ & 0.113 & $7.9(-2.0,17.7)$ & 0.118 & $1.8(-10.0,13.7)$ & 0.759 \\
\hline 5 & 30 & $62.5(16.5)$ & 0.0 & & 0.0 & & 0.0 & \\
\hline Total & 149 & $69.6(17.9)$ & & & & & & \\
\hline
\end{tabular}

A. Mean pressure in $\mathrm{mm} \mathrm{Hg}$ with standard deviation by childhood and adult height fifths.

B. Mean difference in pressure compared with tallest fifth using multiple linear regression, adjusted for sex, age at interview, BMI, and use of antihypertension medication. Childhood and adult heights are modelled separately.

C. As model B, but with additional adjustment for childhood symptom score, childhood BMI, family expenditure on food, household crowding, father's class, number of years smoking, number of years in a job with low decision latitude, number of benefits being received, smoking status, car ownership, and social class. Childhood and adult heights are modelled separately.

D. As model C, but childhood and adult heights are included in the same model.

3). The shortest height fifth is associated with a disproportionally high systolic blood pressure and pulse pressure. Sex standardised adult height shows a weaker relation with systolic blood pressure and pulse pressure. The lowest height fifths (childhood and adult heights) are significantly associated with higher systolic blood pressure and pulse pressure after adjustment for sex, age at interview, adult BMI, and current use of antihypertension medication. Exclusion of those on antihypertensive medication $(n=39)$ did not significantly alter any of the results reported here.

The relations of height with blood pressure and pulse pressure remain statisticaly significant after additional adjustment for: childhood symptom score, childhood BMI, family expenditure on food, household crowding, father's class, number of accumulated years of smoking, number of years in a job with low decision latitude, number of benefits being received, smoking status, car ownership, and social class. The relation of the shortest childhood (but not adult) height fifth and higher diastolic blood pressure also attains statistical significance after this additional adjustment.

Where childhood and adult standardised heights were modelled together, in addition to all of the other potential confounding factors, childhood height retained a statistically significant relation with systolic blood pressure and pulse pressure in early old age, while the relation with adult height lost all statistical significance. A similar result was obtained when the two measures of height were modelled as continuous terms. This indicates that growth in childhood rather than fully attained height is most significantly associated with blood pressure.

The univariate relations of accumulated number of years in jobs with low decision latitude with blood pressure and pulse pressure in early old age were not statistically significant $(p>0.1)$. The interaction of the group containing those with the greatest number of years in such jobs with the shortest childhood height fifth after adjustment for the potential confounding factors as well as the main effects (childhood height and decision latitude), was significantly associated with systolic blood pressure and pulse pressure, but not diastolic blood pressure. Those who were both in the shortest childhood height group and had accumulated the largest number of years in a low decision latitude job showed adjusted relative increases of $35.2 \mathrm{~mm} \mathrm{Hg}(6.0,64.4 ; \mathrm{p}=0.019)$ and $25.8 \mathrm{~mm} \mathrm{Hg}(3.5,48.2 ; \mathrm{p}=0.024)$ for systolic blood pressure and pulse pressure, respectively. However, the group containing those in the shortest childhood height fifth who had also accumulated the greatest number of years in a low decision latitude job represents only five individuals, so this result should be treated with caution.

\section{Discussion}

Shorter stature in childhood was significantly associated with higher systolic blood pressure and pulse pressure (as a marker of arterial compliance) in early old age. These relations remain statistically significant after comprehensive adjustment for potential confounding factors throughout life, including adult height.

This study sought to test the hypothesis that slow growth is a risk for higher blood pressure. Growth was estimated by including standardised childhood and adult heights in the same model. The well established technique is known as conditional change modelling, ${ }^{20}$ as relations with one measure are conditioned on a second. Significant associations with shorter childhood height but not adult height, when both measures are included in the same model, indicate that this is not a function of height per se, but that shorter childhood stature relative to adult height is important: this represents growth. ${ }^{45} \mathrm{~A}$ high proportion of shorter children will continue growing and achieve a relatively taller adult height through later catch up growth. ${ }^{21}$ Examining childhood stature while adjusting for fully attained adult height also represents a powerful adjustment for genetically determined height. ${ }^{4}$ 
Furthermore, this form of analysis will result in conservative estimates of relations with childhood height (therefore growth), as risk factors which influence childhood growth, may also reduce final adult height, despite catch up growth.

Differentials in blood pressure widen over life, ${ }^{22}$ possibly amplifying the effect of exposures in earlier life. Cardiovascular morbidity and mortality associated with higher blood pressure are more likely to be observed in old age, so this period is of particular interest. Environmental risks accumulated across the life course $\mathrm{e}^{23}$ may be important in influencing blood pressure, so statistical adjustment was made for these. The relation of childhood stature with blood pressure is independent of potentially confounding material circumstances, suggesting that these are relatively unimportant, or that growth rate is a more sensitive measure of material circumstances.

This study indicates that growth rate is more strongly associated with blood pressure than fully attained height. Slow growth itself may influence blood pressure, or subsequent catch up growth could represent a risk. It is unlikely that the relation with blood pressure is solely a result of shorter children being of lower birth weight, as it has been shown that the relation of childhood height with blood pressure is independent of birth weight. ${ }^{1}$ Growth rate is strongly influenced by childhood environmental factors, ${ }^{14}$ and the relation between psychosocial stress and slow growth is independent of birth weight. ${ }^{4}$ Growth, rather than BMI or weight gain, appears to be the most important indicator of risk for higher blood pressure, as the relation is independent of childhood and adult BMI.

The a priori hypothesis that resulted in this work being conducted was that psychosocial stress could slow growth and also impair the negative feedback mechanism for the stress response, therefore increasing the risk of higher blood pressure. Childhood stress has been shown to slow growth independent of material and dietary factors, ${ }^{4} 7$ and early stress may also reduce the number of hippocampal glucocorticoid receptors that are expressed, resulting in poorer negative feedback sensitivity for the stress response. ${ }^{89}$ As psychosocial stress has been linked with an increased risk for higher blood pressure, ${ }^{24}{ }^{25}$ poorer control of the stress response is likely to increase the risk for hypertension. Stress in early life may slow growth as well as increasing an individual's vulnerability to subsequent stress and therefore higher blood pressure. This process may operate throughout childhood, but it is likely to begin in utero. There is a positive correlation between maternal and foetal cortisol concentrations, ${ }^{26}$ suggesting that maternal psychosocial stress may also be important in programming the stress response and influencing growth: birth weight is associated with subsequent plasma cortisol concentration ${ }^{27}$ and blood pressure. ${ }^{28}$ The statistically significant increased risk of higher blood pressure associated with low decision latitude at work only among the slowest growing individuals is consistent with the hypothesis that slow growth is a marker of vulnerability to stress, but the small sample size prevents a definitive conclusion from being drawn.

Environmental exposures during periods of rapid growth are likely to be particularly important in influencing subsequent blood pressure. This may be related to growth itself or because slow growth is an indicator of exposures that also influence blood pressure control mechanisms, but these explanations may not be mutually exclusive. Risks accumulated throughout life will determine who becomes hypertensive in later life, but early exposures could increase vulnerability to later risks.

This research was funded through a grant from the Economic and Social Research Council (ESRC).

1 Whincup PH, Cook DG, Adshead F, et al. Cardiovascular risk factors in british children from towns with widely differing adult cardiovascular mortality. BMF 1996;313:7984 .

2 Hense HW, Steiber J, Keil U. The association of short stature with systolic blood pressure, pulse pressure and isolated systolic hypertension. Circulation 1996;93:p31.

3 Smulyan H, Marchais SJ, Pannier B, Guerin AP, Safar ME, London GM. Influence of body height on pulsatile arterial hemodynamic data. f Am Coll Cardiol 1998;31: 1103-9.

4 Montgomery SM, Bartley MJ, Wilkinson RG. Family conflict and slow growth. Arch Dis Child 1997;77:32630.

5 Montgomery SM, Bartley MJ, Cook DG, Wadsworth MEJ. Health and social precursors of unemployment in young men in Great Britain. $\mathcal{F}$ Epidemiol Community Health 1996; 50:415-22.

6 Lever AF, Harrap SB. Essential hypertension: a disorder of growth with origins in childhood? $\mathcal{F}$ Hypertens 1992;10: 101-20.

7 Widdowson EM. Mental contentment and physical growth. Lancet 1951;i:1316-18.

8 Liu D, Diorio J, Tannenbaum B, et al. Maternal care, hippocampal glucocorticoid receptors, and hypothalamicpituitary-adrenal responses to stress. Science 1997;277: 1659-62

9 Sapolsky RM. The importance of a well-groomed child. Science 1997;277:1620-1.

10 Hallqvist J, Diderichsen F, Theorell T, Reuterwall C, Ahlbom A. Is the effect of job strain on myocardial infarction risk due to interaction between high psychological demands and low decision latitude? Results from Stockholm heart epidemiology program (SHEEP). Soc Sci Med 1998;46:1405-15.

11 Bosma H, Marmot MG, Hemingway H, Nicholson AC, Brunner E, Stansfeld S. Low job control and risk of coronary heart disease in Whitehall II (prospective cohort) study. ary heart disease in Whit

12 Alterman T, Shekelle RB, Vernon SW, Burau KD. Decision latitude, psychologic demand, job strain, and coronary heart-disease in the Western Electric study. Am $\mathcal{F}$ Epidemiol 1994;139:620-7

13 Rowett Research Institute. Family diet and health in pre-war Britain. Dunfermline: Carnegie United Kingdom Trust, 1955 .

14 Blane D, Berney LR, Smith GD, Gunnell DJ, Holland P. Reconstructing the life course: health during early old age in a follow-up study based on the Boyd Orr cohort. Public Health 1999;113:117-24.

15 Gunnell DJ. Food, death and income-a follow-up study based on a survey of family diet and health in pre-war Britain (1937-39). PhD thesis, University of Bristol, 1996.

16 Gunnell DJ, Frankel S, Nachahal K, Braddon F, Davey Smith G. Lifecourse exposure and later disease: a follow-up study based on a survey of family diet and health in pre-war Britain (1937-9). Public Health 1996;110: $85-94$.

17 Berney LR, Blane D. Collecting retrospective data: accuracy of recall after 50 years judged against historical records. Soc Sci Med 1997;45:1519-25.

18 Office for National Statistics. 1991 Census. London: The Stationary Office, 1993.

19 Gunnell DJ, Davey Smith G, Frankel S, et al. Childhood leg length and adult mortality: follow up of the Carnegie (Boyd Orr) survey of diet and health in pre-war Britain. $f$ Epidemiol Community Health 1998;52:142-52.

20 Plewis I. Analysing change. London: Wiley, 1985.

21 Greco L, Power C, Peckham C. Adult outcome of normal children who are short or underweight at age 7 years. $B M F$ 1995;310:696-700. 
22 de Simone G, Roman MJ, Daniels SR, et al. Age-related changes in total arterial capacitance from birth to maturity in

23 Pickering TG, Devereux RB, James GD, et al. Environmental influences on blood pressure and the role of job strain. $f$ Hypertens 1996;14:s179-85.

24 Steptoe A, Cropley M, Joekes K. Job strain, blood pressure and response to uncontrollable stress. $\mathcal{F}$ Hypertens $1999 ; 17$ : 193-200.

25 Pickering T. The effects of occupational stress on blood pressure in men and women. Acta Physiol Scand 1997;161:125-8.
26 Gitau R, Cameron A, Fisk NM, Glover V. Fetal exposure to maternal cortisol. Lancet 1998;352:707-8.

27 Phillips DIW, Barker DJP, Fall CHD, et al. Elevated plasma cortisol concentrations: a link between low birth weight and the insulin resistance syndrome? $\mathcal{F}$ Clin Endocrinol Metab 1998;83:757-60.

28 Nilsson PM, Ostergren PO, Nyberg P, Soderstrom M, A llebeck P. Low birth weight is associated with elevated systolic blood pressure in adolescence: a prospective study of a birth cohort of 149,378 Swedish boys. F Hypertens 1997; 15:1627-31.

\section{Rapid responses}

If you have a burning desire to respond to a paper published in $A D C$ or $F \mathcal{E} N$, why not make use of our "rapid response" option?

Log on to our website (www.archdischild. com), find the paper that interests you, click on "abstract" or "full text" and send your response by email by clicking on "submit a response".

Providing it isn't libellous or obscene, it will be posted within seven days. You can retrieve it by clicking on "read rapid responses" on our homepage.

The editors will decide, as before, whether to also publish it in a future paper issue. 